\title{
Lung Ultrasound and Respiratory Pathophysiology in Mechanically Ventilated COVID-19 Patients—an Observational Trial
}

\author{
Marco Rojatti ${ }^{1}$ - Ivo B. Regli ${ }^{2,3}$ - Alessandro Zanforlin ${ }^{4}$ Enrica Ferretti ${ }^{1} \cdot$ Markus Falk $^{3}$ • Giacomo Strapazzon ${ }^{3,5}$. \\ Magdalena Gamper ${ }^{2,6} \cdot$ Peter Zanon ${ }^{1} \cdot$ Matthias Bock ${ }^{2,7} \cdot$ Simon Rauch ${ }^{2,3}$ (I)
}

Accepted: 21 September 2020 / Published online: 25 September 2020

(C) Springer Nature Switzerland AG 2020

\begin{abstract}
The relationship between respiratory system mechanics, lung ultrasound (LUS) abnormalities, and mortality in mechanically ventilated patients with COVID-19-associated respiratory failure is unknown. We assessed the pattern of respiratory mechanics and LUS, their changes over time, and the differences between survivors and non-survivors. We additionally analyzed the relationship between LUS findings and the severity of gas exchange impairment and interleukin 6 (IL-6). This was a twocenter retrospective, observational trial carried out in the intensive care units of the hospitals of Bolzano and Merano, Italy, from March 15 to April 20, 2020. We enrolled 41 consecutive patients. Seven patients (17\%, 95\% CI 8.5-31.3\%) died. Mean compliance of the respiratory system on ICU admission was $41.6( \pm 18.8) \mathrm{ml} / \mathrm{mbar}(42.5( \pm 19.6)$ for survivors, $38.0( \pm 16.3)$ for deceased, $p=0.605$ ). Non-survivors had a significantly lower compliance over time, decreasing from day 14 after symptom onset, compared with survivors $(p=0.008)$. Mean LUS score on admission was $11.2( \pm 3.7)$ and survivors had lower LUS scores on admission than non-survivors (10.5 $( \pm 3.6), 13.9(2.8)$, respectively, $t$ test, $p=0.029)$. LUS score correlated with IL-6 concentrations $(r=0.52, p=0.001)$ and arterial $\mathrm{pCO}_{2}(r=0.30, p=0.033)$ and was inversely correlated with oxygenation $(r=$ $-0.34, p=0.001)$. No correlation was found between LUS and respiratory system compliance $(r=-0.02, p=0.299)$. Nonsurvivors from COVID-19-associated respiratory failure had a significant decrease in compliance after day 14 of symptom onset. Compliance did not correlate with the degree of abnormalities found in LUS, but LUS score correlated with oxygenation, $\mathrm{pCO}_{2}$, and IL-6.
\end{abstract}

Keywords COVID-19 $\cdot$ ARDS $\cdot$ Mechanical ventilation $\cdot$ Lung ultrasound $\cdot$ Respiratory mechanics

\section{Background}

A new form of viral pneumonia caused by the severe acute respiratory syndrome coronavirus 2 (SARS-CoV-2) was identified in Wuhan, China, in December 2019 [1-3]. Due to its transmission dynamics, the virus spread quickly across the globe within a very short time and on March 11, 2020, the WHO declared the outbreak a pandemic [4]. Studies from
Marco Rojatti and Ivo B. Regli contributed equally to this work.

This article is part of the Topical Collection on COVID-19

Simon Rauch

simon.rauch@sabes.it

1 Department of Anesthesiology and Intensive Care, "S. Maurizio" Hospital, Via Lorenz Boehler 5, 39100 Bolzano, Italy

2 Department of Anesthesiology and Intensive Care, "F. Tappeiner" Hospital, Via Rossini 5, 39012 Merano, Italy

3 Institute of Mountain Emergency Medicine, Eurac Research, Viale Druso 1, 39100 Bolzano, Italy
4 Servizio Pneumologico Aziendale, Azienda Sanitaria dell'Alto Adige, Via Lorenz Boehler 5, 39100 Bolzano, Italy

5 Department of Anesthesiology and Intensive Care Medicine, Medical University Innsbruck, Anichstraße 35, 6020 Innsbruck, Austria

6 Medical University of Vienna, Medicine Degree Program, Spitalgasse 23, 1090 Vienna, Austria

7 Department of Anesthesiology, Perioperative Medicine and Intensive Care, Paracelsus Medical University, Strubergasse 21, 5020 Salzburg, Austria 
China reported a high incidence of critical illness (23-32\%) and acute respiratory distress syndrome (ARDS) (17-29\%) among hospitalized patients [1, 5-7].

The pathophysiology of coronavirus disease 2019 (COVID-19)-associated respiratory failure is still the subject of debate and ongoing research. Even though most COVID19 patients needing mechanical ventilation fulfil the Berlincriteria [8], severe respiratory failure due to COVID-19 pneumonia is distinct from classical ARDS and (at least at the early stage of the disease) characterized by a dissociation between relatively well-preserved lung mechanics and the severity of hypoxemia. Gattinoni et al. suggested that two COVID-19 phenotypes can be distinguished: type L, characterized by a maintained lung compliance, near normal lung weight, and low lung recruitability, and type $\mathrm{H}$, characterized by a poor lung compliance, an increase in lung weight, and high lung recruiting capacity [9]. Type $\mathrm{L}$ can progress to type $\mathrm{H}$ as the disease progresses and depending on the phenotype, different treatment strategies might be adapted [9]. Yet, further evidence is needed to prove this hypothesis. To date, little is known about the changes of respiratory system compliance over time in mechanically ventilated COVID-19 patients and the relationship between respiratory system mechanics and mortality.

Lung ultrasonography (LUS) allows rapid and reliable diagnosis of interstitial-alveolar syndrome, lung consolidation or pleural effusion, and is superior to chest radiography [10]. When evaluating patients with pneumonia and/or ARDS, LUS gives results similar to chest CT [11]. During the COVID-19 pandemic, point of care LUS has been widely adopted and characteristic findings of COVID-19 pneumonia have been described [12-15]. However, little is known about the relationship between LUS findings and the severity of gas exchange impairment and between LUS and lung mechanics in COVID-19 pneumonia. Moreover, it is unknown whether LUS abnormalities are linked to the degree of systemic inflammation (e.g., assessed by interleukin 6 (IL-6) plasma concentration) or to mortality.

Our study aimed at assessing the pattern of respiratory mechanics and LUS in mechanically ventilated patients with COVID-19-associated respiratory failure, their changes over time, and the differences between survivors and non-survivors. We additionally analyzed the relationship between LUS findings and the severity of gas exchange impairment and IL-6.

\section{Methods}

In this observational trial, the medical records of consecutive patients admitted to the intensive care units (ICUs) of the hospitals of Bolzano and Merano, South Tyrol, Italy, from March 15 to April 20, 2020, due to COVID-19-associated respiratory failure were retrospectively analyzed. The study was approved by the Ethics Committee of South Tyrol (protocol number 46-2020). Study design and reporting were performed according to the Strengthening the Reporting of Observational Studies in Epidemiology (STROBE) statement [16].

\section{Clinical Management}

All patients were treated at the discretion of the attending physicians. COVID-19-associated respiratory failure was managed according to the current guidelines [17], including lung protective ventilation, prone positioning, use of neuromuscular blocking agents (if indicated), and a conservative fluid balance. Laboratory tests were performed according to the clinical indications and protocols in use in each institution.

\section{Data Collection}

Ventilatory parameters, LUS data, and laboratory values were routinely recorded in the ICU patient data managing system. The following data were extracted and stored anonymously: sex, age, mode of ventilation, PEEP, driving pressure, respiratory system compliance, $\mathrm{PaO}_{2}, \mathrm{FiO}_{2}, \mathrm{PaCO}_{2}$, IL-6 plasma concentration (Elecsys $\odot$ IL-6, Roche Diagnostics, Espoo, Finland), LUS score, ICU length of stay (ICU-LOS), and outcome (alive or deceased). Respiratory system compliance was calculated in patients under controlled mechanical ventilation as tidal volume (TV) / (plateau pressure - PEEP).

\section{Lung Ultrasound}

The scan was performed with the patient in supine position. A convex probe with abdominal preset was selected, without compound and with general frequency setting. Focus was set at the pleural line, depth at $6-8 \mathrm{~cm}$, and gain low (i.e., to see pleural sliding). The two ultrasound scanners used (MyLab30 Esaote spa, Genova, Italy, and X-porte Fujifilm-Sonosite Inc., Amsterdam, The Netherlands) were dedicated exclusively to COVID-19 patients. The probe and hardware were cleaned after each use according to the local protocols. In order to obtain the LUS score, the surface of each hemithorax was divided into four lung fields [18]. For each lung field, a numerical score was given: 0 for A lines, 1 for well separated B lines, 2 for coalescent $\mathrm{B}$ lines, and 3 for the presence of parenchymal consolidations (aerial bronchogram or atelectasis). We used the sum of the score assigned to each lung field on both sites (i.e., maximal score of 24 points) for analysis. The LUS examination was done routinely and not driven by a clinical indication (e.g., a deterioration of gas exchange). 


\section{Statistical Analysis}

We presented data as percentages for counted and as mean and standard deviation (SD) for numerical data. IL-6 values were $\log$ transformed to achieve normality prior to analysis. Group comparisons for baseline values or mean values over all time points were carried out by means of the Chi-square test or the t-test. Linear mixed models (LMM) were fitted to the data when utilizing all time points using the patient as a random effect with a random intercept and the fixed effects status, hospital, time (grouped in quintiles) and the covariate LUS. Additionally, correlation coefficients between continuous variables have been assessed by means of the partial correlation coefficient controlling for patient and hospital. For analysis of changes over time, the day of symptom onset rather than the timepoint of ICU admission was considered as baseline (i.e. day 1). All $p$ values are twosided and values below 0.05 have been considered significant. SPSS (IBM version 26.0) was used for statistical analysis.

\section{Results}

\section{Patient Characteristics}

We included 41 consecutive patients. All patients were intubated and mechanically ventilated on ICU admission. Table 1 summarizes patients' demographics, comorbidities, respiratory parameters, and laboratory values on admission, divided between survivors and non-survivors.

Table 1 Baseline demographic characteristics, comorbidities, respiratory parameters, and laboratory values, divided between survivors and nonsurvivors. We revealed these data on admission

\begin{tabular}{|c|c|c|c|c|}
\hline & Survivors $n=34(82 \%)$ & Non-survivors $n=7(17 \%)$ & Overall $n=41$ & $p$ value \\
\hline Age - mean (SD) & $59.4(11.8)$ & $73(5.1)$ & $61.7(12.1)$ & $0.005^{*}$ \\
\hline \multicolumn{5}{|l|}{ Sex-no. $(\%)$} \\
\hline Male & $26(81 \%)$ & $6(19 \%)$ & $32(78 \%)$ & \multirow[t]{2}{*}{$0.591^{+}$} \\
\hline Female & $8(89 \%)$ & $1(11 \%)$ & $9(22 \%)$ & \\
\hline $\mathrm{BMI}$-mean $(\mathrm{SD})$ & $27.1(4.3)$ & $25.3(5)$ & $26.7(4.4)$ & $0.335^{*}$ \\
\hline \multicolumn{5}{|l|}{ Enrolment site } \\
\hline Bolzano-no. $(\%)$ & $15(79 \%)$ & $4(21 \%)$ & $19(46 \%)$ & \multirow[t]{2}{*}{$0.529^{+}$} \\
\hline Merano-no. (\%) & $19(86 \%)$ & $3(14 \%)$ & $22(54 \%)$ & \\
\hline \multicolumn{5}{|l|}{ Comorbidities - no. (\%) } \\
\hline Arterial hypertension & & & $13(32 \%)$ & \\
\hline Coronary artery disease & & & $1(2 \%)$ & \\
\hline Atrial fibrillation & & & $2(5 \%)$ & \\
\hline Diabetes & & & $5(12 \%)$ & \\
\hline COPD & & & $4(10 \%)$ & \\
\hline Chronic kidney disease & & & $5(12 \%)$ & \\
\hline History of smoking & & & $5(12 \%)$ & \\
\hline Obesity & & & $5(12 \%)$ & \\
\hline History of malignancy & & & $7(17 \%)$ & \\
\hline None & & & $11(27 \%)$ & \\
\hline Time (days) from symptom onset to ICU admission — mean (SD) & $9.8(3.7)$ & $12.8(9.8)$ & $10.2(5)$ & $0.167 *$ \\
\hline Length of ICU stay — days, mean (SD) & $17.8(7.7)$ & $14.9(10.5)$ & $17.3(8.2)$ & $0.399 *$ \\
\hline Number of prone positionings during ICU stay - mean (SD) & $1.9(1.8)$ & $2.0(1.4)$ & $1.9(1.7)$ & $0.891^{*}$ \\
\hline $\mathrm{PaO}_{2} / \mathrm{FiO}_{2}$ on day 1 of $\mathrm{ICU}$ admission - mean $(\mathrm{SD})$ & $183.1(71)$ & $185.3(61.3)$ & $183.4(68.7)$ & $0.940^{*}$ \\
\hline $\mathrm{PaO}_{2}$ on day 1 of $\mathrm{ICU}$ admission — mean $(\mathrm{SD})$ & $90.4(24.2)$ & $77.2(22.7)$ & $88.4(24.2)$ & $0.221 *$ \\
\hline $\mathrm{PaCO}_{2}$ on day 1 of ICU admission - mean (SD) & $46.9(15.5)$ & $41.1(11)$ & $46(14.9)$ & $0.394 *$ \\
\hline Respiratory system compliance on day $1-\mathrm{ml} / \mathrm{mbar}$, mean (SD) & $42.5(19.6)$ & $38.0(16.3)$ & $41.6(18.8)$ & $0.605^{*}$ \\
\hline PEEP on day 1 of ICU admission — mbar, mean (SD) & $13.3(2.2)$ & $13.7(1.9)$ & $13.3(2.2)$ & $0.625^{*}$ \\
\hline IL-6 plasma concentration on admission — pg/ml, mean (SD) & $530.8(1545)$ & $552.9(763.7)$ & $534.3(1433.2)$ & $0.304 *$ \\
\hline
\end{tabular}




\section{Respiratory System Compliance}

Mean respiratory system compliance on day 1 of ICU admission was $42.5( \pm 19.6) \mathrm{ml} / \mathrm{mbar}$ for survivors and $38.0( \pm 16.3)$ $\mathrm{ml} / \mathrm{mbar}$ for non-survivors. We found no significant difference between survivors and non-survivors on admission $(t$ test, $p=0.605$ ) or as mean over all time points ( $t$ test, $p=$ 0.066 , Fig. 1a). However, when comparing compliance over time, we revealed that non-survivors had a significantly lower compliance from day 14 after symptoms onset compared with surviving patients ( $p=0.008$; Fig. $1 b)$.

\section{Lung Ultrasonography}

Of the 41 patients included in this study, 34 underwent regular LUS examinations with a median of 5 LUS exams per patient during the ICU stay (i.e., an exam about every $72 \mathrm{~h}$ ). Mean LUS score on admission was $11.2( \pm 3.7)$ and was significantly different between survivors and non-survivors $(10.5( \pm 3.6)$,
13.9 (2.8), respectively, $t$ test, $p=0.029$ ). Non-survivors had higher but not significantly different mean LUS scores over all time points than survivors $(12.9 \pm 4.6,9.9 \pm 3.4$, respectively, $t$ test, $p=0.061$, Fig. 2a). In contrast to the respiratory system compliance, there was no time-dependent effect when comparing these two groups (LMM status effect, $p=0.141$, Fig. $2 b$ ).

We found a significant inverse correlation between the LUS score and the $\mathrm{PaO}_{2} / \mathrm{FiO}_{2}$ ratio $(r=-0.34, p=0.001$, Fig. 3a). Furthermore, we found a significant correlation between the LUS score and $\mathrm{PaCO}_{2}(r=0.30, p=0.033$, Fig. $3 b)$ and a significant correlation between LUS score and IL-6 plasma levels $(r=0.52, p=0.001$, Fig. 3c). We found no correlation when comparing LUS and compliance $(r=-0.02, p=0.299)$.

\section{Discussion}

In our study, we describe a series of 41 consecutive, mechanically ventilated patients with COVID-19-associated
Fig. 1 Respiratory system compliance values of mechanically ventilated patients with COVID-19-associated respiratory failure. a Mean compliance values during ICU stay of survivors and non-survivors. Box lines represent the 25th and 75th percentile. b Compliance values of survivors and non-survivors over time a

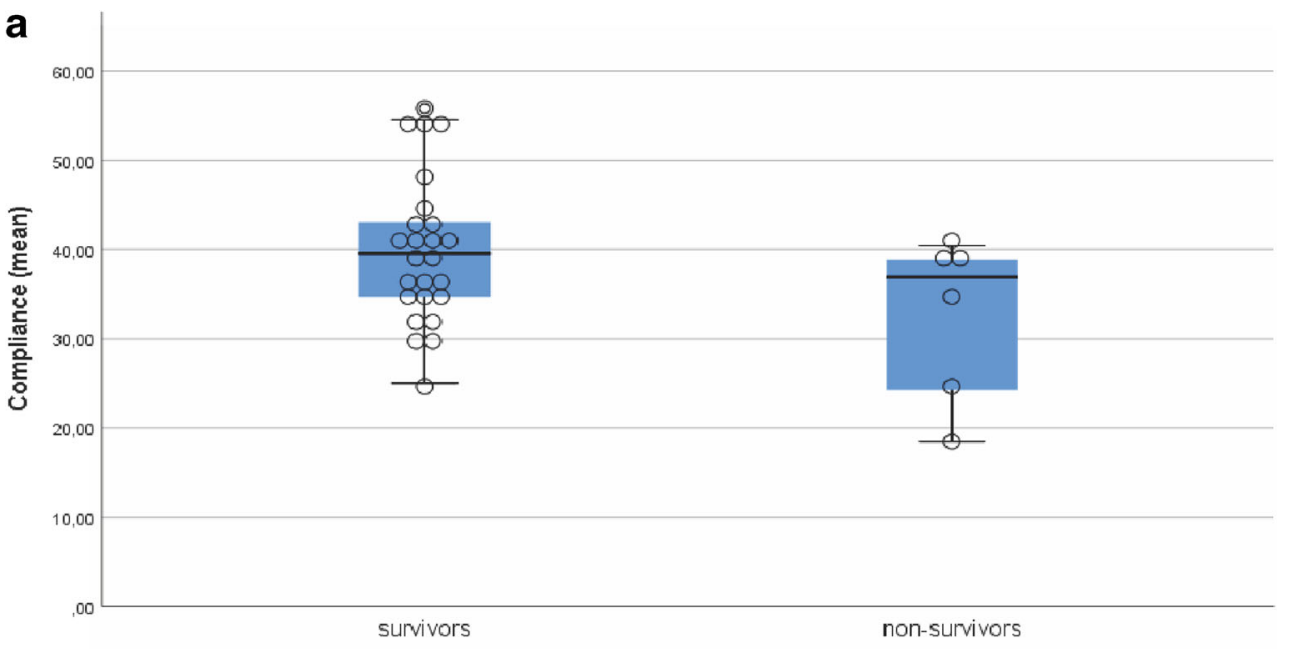

b
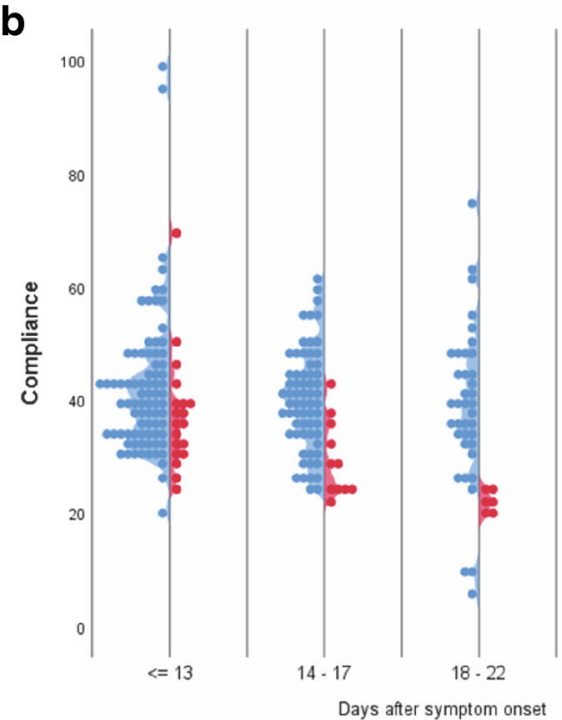

- survivors

non-survivors 
Fig. 2 LUS score of mechanically ventilated patients with COVID-19-associated respiratory failure. a Mean LUS score during ICU stay of survivors and non-survivors. Box lines represent the 25th and 75th percentile. b LUS score of survivors and non-survivors over time. $n=34$ a

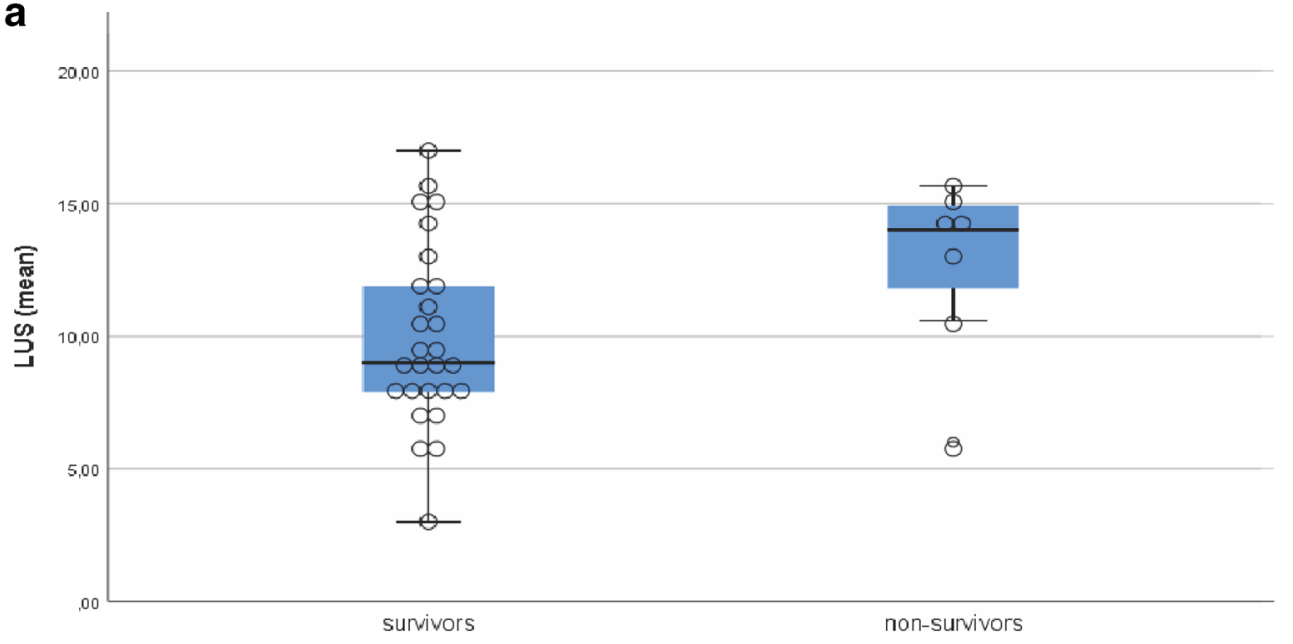

b

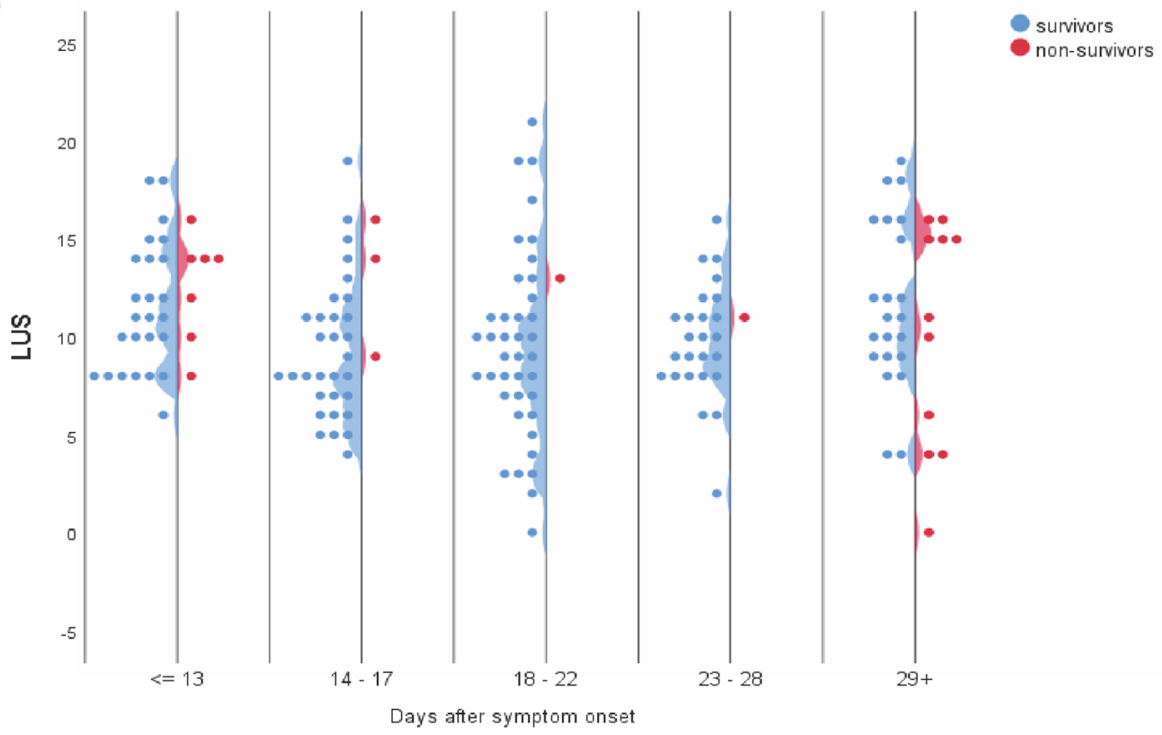

respiratory failure. Overall mortality rate was $17 \%$ (95\% CI 8.5-31.3\%) with non-survivors being significantly older than survivors. We found that mechanically ventilated ICU patients not surviving COVID-19 had a lower compliance after 2 weeks from symptom onset and a higher LUS score compared with survivors. Moreover, we showed that LUS score is a potential indicator of respiratory function and inflammatory status, as it correlates with gas exchange and IL-6 plasma concentration.

COVID-19-associated respiratory failure differs from classical ARDS. Though the Berlin ARDS criteria [8] are often met at presentation, a number of characteristics are atypical, such as a near normal lung volume with normal respiratory mechanics and compliance [9]. Gattinoni et al. named this initial presentation phenotype L. Yet, some patients undergo transition from phenotype $\mathrm{L}$ to phenotype $\mathrm{H}$, the latter resembling classical ARDS with reduced lung volume, increased lung weight, and decreased compliance [9]. However, more than two phenotypes, type $\mathrm{L}$ and $\mathrm{H}$ might reflect two stages of the disease: COVID-19 pneumonia can over time develop classical ARDS, like any pneumonia caused by other pathogens [19]. This is in line with our findings, showing that some patients continue to have normal lung mechanics while others develop a reduced compliance approximately 2 weeks after symptom onset. This time point corresponds to about day 4 of ICU admission as mean time from symptom onset to ICU admission in our cohort is 10 days. Expectedly, patients with a decrease in lung compliance over time, i.e., patients developing classical ARDS, have a higher mortality rate than patients with preserved lung mechanics, i.e., patients with COVID-19 pneumonia without classical ARDS. Our findings on temporal changes in lung mechanics and their relationship to disease severity are in agreement with changes in CT findings over time found by Li et al. [20]. They described that pulmonary lesions with extensive ground-glass opacities and consolidations generally peaked around 14 days after the onset of the disease. 

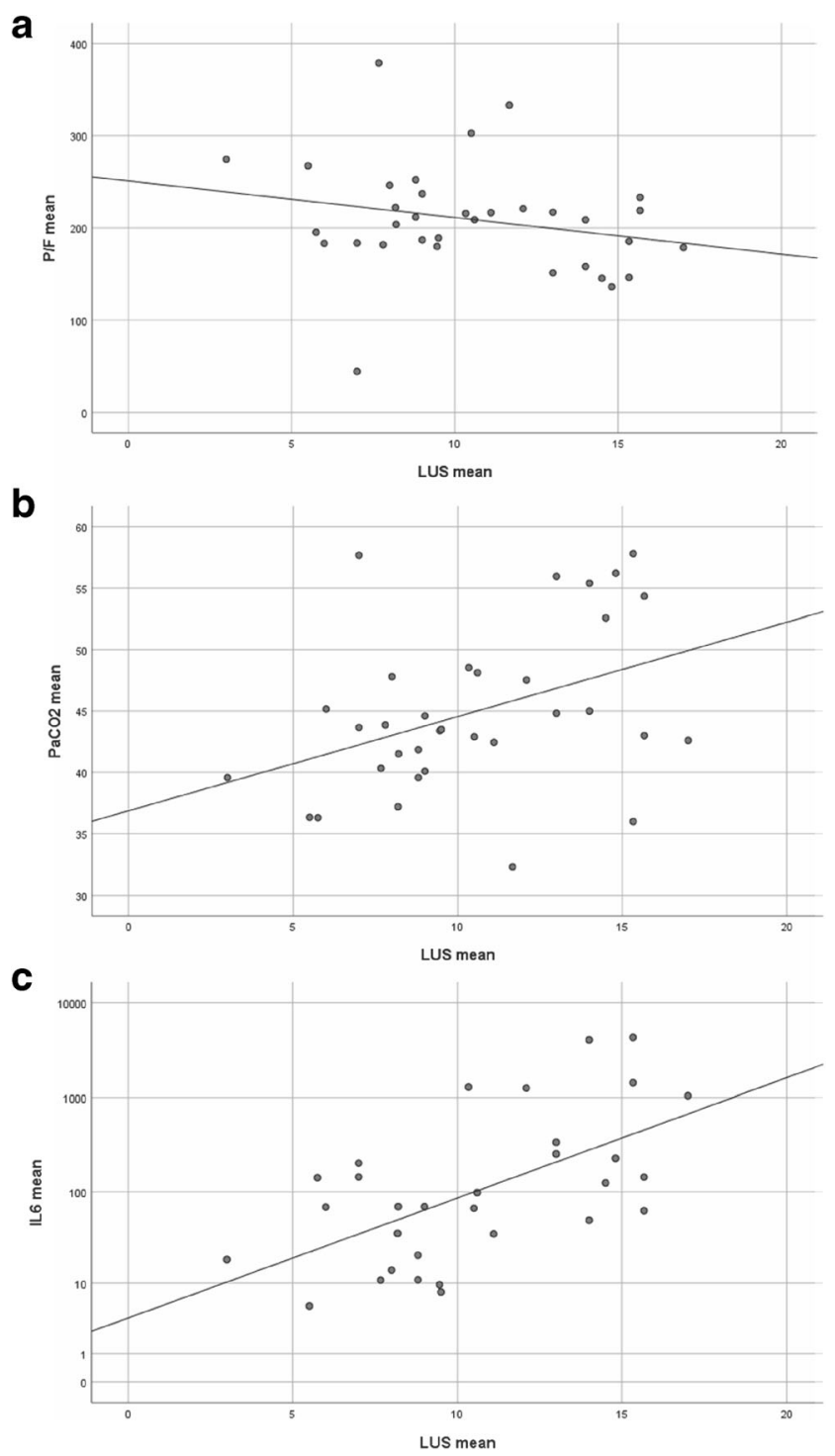

Fig. 3 Correlation of LUS score with respiratory and inflammatory parameters. a Scatter plot of LUS score versus $\mathrm{PaO}_{2} / \mathrm{FiO}_{2}$ ratio. b Scatter plot of LUS score versus $\mathrm{PaCO}_{2}$. c Scatter plot of LUS score versus IL-6 plasma concentration. Points represent respective mean values over all time points for one patient and regression lines are indicated. IL-6 plasma concentration depicted in logarithmic $\left(\log _{10}\right)$ scale. $n=34$

Chest CT scan is the gold standard imaging modality for pneumonia and ARDS [21]. CT scan is not universally available during a pandemic of a highly infectious disease with a large number of patients requiring hospital and intensive care unit admission. LUS, on the other hand, is a nearly universally available, a highly reproducible bedside technique with a short learning curve, and a reliable tool to identify interstitial syndrome, lung consolidation, lung collapse, and pleural effusion [10, 22-26]. Recently, its utility for diagnosis and follow-up of patients with COVID-19 pneumonia has been shown [12, $27,28]$. We found a significant correlation between the degree of abnormality in LUS (expressed as LUS score) and abnormalities in pulmonary gas exchange: The higher the LUS score, the lower the $\mathrm{PaO}_{2} / \mathrm{FiO}_{2}$ ratio and the higher the $\mathrm{PaCO}_{2}$. Thus, the LUS findings of a severe interstitial syndrome and/or of consolidations are predictive of a ventilation/perfusion mismatch with shunt and dead space ventilation, leading to hypoxemia and hypercapnia. This correlation between LUS and morphologic and functional lung alterations was reflected on survival: LUS score on admission was significantly higher in nonsurvivors compared with survivors (and marginally significantly higher over time). On the other hand, we did not find a correlation between LUS score and respiratory system compliance. This is in contrast to chest CT scan, where the size of the so called "baby lung" in an ARDS patients correlates with compliance [29]. There are several potential limitations concerning the evaluation of lung mechanics with ultrasound that could explain our findings, e.g., the limited penetration of ultrasound waves into the lung parenchyma and the related difficulty to assess lung volume.

We found a correlation between LUS score and the plasma concentration of IL-6. IL-6 is a cytokine with a wide variety of context-dependent effects on immunity and inflammation that seems to be an important factor in the COVID-19 pathogenesis [30, 31]. Increased IL-6 plasma levels correlated with morbidity and mortality in COVID19 patients [32, 33]. Furthermore, this cytokine might be directly involved in the pathogenesis of acute lung injury, presumably via recruitment of inflammatory cells in the lung [34-36]. It is a hallmark cytokine released during macrophage activation syndrome and cytokine storm, which are both potential key processes in COVID-19 disease exacerbation [37]. In contrast to other studies, we found no difference in IL-6 plasma concentration between survivors and non-survivors on admission. We observed, however, a correlation between LUS score and plasma IL-6 concentration during the time course of the disease, underlining the role of this cytokine in the pathogenesis and the severity of COVID-19 pneumonia. LUS score might therefore reflect the degree of inflammatory damage in the lung parenchyma. Yet, this issue remains object of further investigations.

\section{Limitations}

The retrospective design of the study and the limited number of patients are the main limitations of our study. LUS is an operator dependent technique, but during the emergency related to the pandemic, it was not possible to assess the interoperator agreement. However, only experienced clinicians performed LUS using already tested international protocols. 


\section{Conclusion}

In this series of mechanically ventilated COVID-19 patients, respiratory system compliance differed between survivors and non-survivors with the latter having a significant decrease in compliance after day 14 of symptom onset. Compliance did not correlate with the degree of abnormalities found in LUS, but LUS score correlated with survival, oxygenation, ventilation, and IL-6. LUS gives a dynamic bed-side view of the respiratory function and pathophysiology in patients with COVID-19-associated respiratory failure.

Authors' Contribution MR, IR, MF, and SR had full access to all the data in the study and take responsibility for the integrity and the accuracy of the data analysis. MR, IR, GS, and SR conceived the study. MR, IR, EF, $\mathrm{AZ}$, and SR collected the data for the study. AZ, EF, PZ, MG, and MB participated in analysis and interpretation of data, helped to draft the manuscript, and critically revised the manuscript for important intellectual content. MF was in charge of the statistical analysis. All authors read and approved the final manuscript.

\section{Compliance with Ethical Standards}

The study was approved by the Ethics Committee of South Tyrol (protocol number 46-2020). Study design and reporting were performed according to the Strengthening the Reporting of Observational Studies in Epidemiology (STROBE) statement [16].

Conflict of Interest The authors declare that they have no conflict of interest.

Informed Consent Informed consent was not obtained as data was collected and analyzed in a completely anonymized way.

\section{References}

1. Huang C, Wang Y, Li X, Ren L, Zhao J, Hu Y, et al. Clinical features of patients infected with 2019 novel coronavirus in Wuhan, China. Lancet. 2020;395(10223):497-506. https://doi. org/10.1016/S0140-6736(20)30183-5.

2. Riou J, Althaus CL. Pattern of early human-to-human transmission of Wuhan 2019 novel coronavirus (2019-nCoV), December 2019 to January 2020. Euro surveillance : bulletin Europeen sur les maladies transmissibles. Eur Commun Dis Bull. 2020;25(4). https:// doi.org/10.2807/1560-7917.ES.2020.25.4.2000058.

3. Zhu N, Zhang D, Wang W, Li X, Yang B, Song J, et al. A novel coronavirus from patients with pneumonia in China, 2019. N Engl J Med. 2020;382(8):727-33. https://doi.org/10.1056/ NEJMoa2001017.

4. Mahase E. Covid-19: WHO declares pandemic because of "alarming levels" of spread, severity, and inaction. Bmj. 2020;368:m1036. https://doi.org/10.1136/bmj.m1036.

5. Wang D, Hu B, Hu C, Zhu F, Liu X, Zhang J, et al. Clinical characteristics of 138 hospitalized patients with 2019 novel coronavirus-infected pneumonia in Wuhan, China. JAMA. 2020;323(11):1061-9. https://doi.org/10.1001/jama.2020.1585.

6. Yang X, Yu Y, Xu J, Shu H, Ja X, Liu H, et al. Clinical course and outcomes of critically ill patients with SARS-CoV-2 pneumonia in Wuhan, China: a single-centered, retrospective, observational study. Lancet Respir Med. 2020;8(5):475-81. https://doi.org/10. 1016/S2213-2600(20)30079-5.

7. Chen N, Zhou M, Dong X, Qu J, Gong F, Han Y, et al. Epidemiological and clinical characteristics of 99 cases of 2019 novel coronavirus pneumonia in Wuhan, China: a descriptive study. Lancet. 2020;395(10223):507-13. https://doi.org/10.1016/ s0140-6736(20)30211-7.

8. Force* TADT. Acute respiratory distress syndrome: the Berlin definition. JAMA. 2012;307(23):2526-33. https://doi.org/10.1001/ jama.2012.5669.

9. Gattinoni L, Chiumello D, Caironi P, Busana M, Romitti F, Brazzi L, et al. COVID-19 pneumonia: different respiratory treatments for different phenotypes? Intensive Care Med. 2020;46:1099-102. https://doi.org/10.1007/s00134-020-06033-2.

10. Bouhemad B, Mongodi S, Via G, Rouquette I. Ultrasound for "lung monitoring" of ventilated patients. Anesthesiology. 2015;122(2): 437-47. https://doi.org/10.1097/aln.0000000000000558.

11. Mayo PH, Copetti R, Feller-Kopman D, Mathis G, Maury E, Mongodi S, et al. Thoracic ultrasonography: a narrative review. Intensive Care Med. 2019;45(9):1200-11. https://doi.org/10.1007/ s00134-019-05725-8.

12. Soldati G, Smargiassi A, Inchingolo R, Buonsenso D, Perrone T, Briganti DF, et al. Is there a role for lung ultrasound during the COVID-19 pandemic? J Ultrasound Med. https://doi.org/10.1002/ jum.15284.

13. Peng Q-Y, Wang X-T, Zhang L-N, Chinese Critical Care Ultrasound Study G. Findings of lung ultrasonography of novel corona virus pneumonia during the 2019-2020 epidemic. Intensive Care Med. 2020;46(5):849-50. https://doi.org/10.1007/ s00134-020-05996-6.

14. Buonsenso D, Pata D, Chiaretti A. COVID-19 outbreak: less stethoscope, more ultrasound. Lancet Respir Med. 2020;8(5):e27. https:// doi.org/10.1016/s2213-2600(20)30120-x.

15. Denault AY, Delisle S, Canty D, Royse A, Royse C, Serra XC, et al. A proposed lung ultrasound and phenotypic algorithm for the care of COVID-19 patients with acute respiratory failure. Can J Anaesth. 2020;67:1393-404. https://doi.org/10.1007/s12630-020-01704-6.

16. The PME. Observational studies: getting clear about transparency. PLoS Med. 2014;11(8):e1001711. https://doi.org/10.1371/journal. pmed.1001711.

17. Alhazzani W, Møller MH, Arabi YM, Loeb M, Gong MN, Fan E, et al. Surviving sepsis campaign: guidelines on the management of critically ill adults with coronavirus disease 2019 (COVID-19). Intensive Care Med. 2020;46(5):854-87. https://doi.org/10.1007/ s00134-020-06022-5.

18. Yin W, Zou T, Qin Y, Yang J, Li Y, Zeng X, et al. Poor lung ultrasound score in shock patients admitted to the ICU is associated with worse outcome. BMC Pulm Med. 2019;19(1):1. https://doi. org/10.1186/s12890-018-0755-9.

19. Jain A, Doyle DJ. Stages or phenotypes? A critical look at COVID19 pathophysiology. Intensive Care Med. 2020;46:1494-5. https:// doi.org/10.1007/s00134-020-06083-6.

20. Li M, Lei P, Zeng B, Li Z, Yu P, Fan B, et al. Coronavirus disease (COVID-19): spectrum of CT findings and temporal progression of the disease. Acad Radiol. 2020;27(5):603-8. https://doi.org/10. 1016/j.acra.2020.03.003.

21. Zompatori M, Ciccarese F, Fasano L. Overview of current lung imaging in acute respiratory distress syndrome. Eur Respir Rev. 2014;23(134):519-30. https://doi.org/10.1183/09059180. 00001314.

22. Vetrugno L, Bove T, Guadagnin GM, Orso D, Brussa A, Volpicelli G. Advances in lung ultrasound in critically ill patients. J Emerg Critic Care Med, 2019;3.

23. Wang L, Song W, Wang Y, Han J, Lv K. Lung ultrasonography versus chest radiography for the diagnosis of pediatric community acquired pneumonia in emergency department: a meta-analysis. J 
Thoracic Dis. 2019;11(12):5107-14. https://doi.org/10.21037/jtd. 2019.11.62.

24. Alzahrani SA, Al-Salamah MA, Al-Madani WH, Elbarbary MA. Systematic review and meta-analysis for the use of ultrasound versus radiology in diagnosing of pneumonia. Crit Ultrasound $\mathrm{J}$. 2017;9(1):6. https://doi.org/10.1186/s13089-017-0059-y.

25. Llamas-Alvarez AM, Tenza-Lozano EM, Latour-Perez J. Accuracy of lung ultrasonography in the diagnosis of pneumonia in adults: systematic review and meta-analysis. Chest. 2017;151(2):374-82. https://doi.org/10.1016/j.chest.2016.10.039.

26. Orso D, Guglielmo N, Copetti R. Lung ultrasound in diagnosing pneumonia in the emergency department: a systematic review and meta-analysis. Eur J Emerg Med. 2018;25(5):312-21. https://doi. org/10.1097/MEJ.0000000000000517.

27. Vetrugno L, Bove T, Orso D, Barbariol F, Bassi F, Boero E, et al. Our Italian experience using lung ultrasound for identification, grading and serial follow-up of severity of lung involvement for management of patients with COVID-19. Echocardiography. 2020;37(4):625-7. https://doi.org/10.1111/echo.14664.

28. Smith MJ, Hayward SA, Innes SM, Miller ASC. Point-of-care lung ultrasound in patients with COVID-19 - a narrative review. Anaesthesia. 2020;75:1096-104. https://doi.org/10.1111/anae. 15082.

29. Gattinoni L, Pesenti A, Avalli L, Rossi F, Bombino M. Pressurevolume curve of total respiratory system in acute respiratory failure. Computed tomographic scan study. Am Rev Respir Dis. 1987;136(3):730-6. https://doi.org/10.1164/ajrccm/136.3.730.

30. Hunter CA, Jones SA. IL-6 as a keystone cytokine in health and disease. Nat Immunol. 2015;16(5):448-57. https://doi.org/10.1038/ ni.3153.

31. Magro G. SARS-CoV-2 and COVID-19: is interleukin-6 (IL-6) the 'culprit lesion' of ARDS onset? What is there besides Tocilizumab?
SGP130Fc. Cytokine X. 2020. https://doi.org/10.1016/j.cytox. 2020.100029 .

32. Zhou F, Yu T, Du R, Fan G, Liu Y, Liu Z, et al. Clinical course and risk factors for mortality of adult inpatients with COVID-19 in Wuhan, China: a retrospective cohort study. Lancet. 2020;395(10229):1054-62. https://doi.org/10.1016/S01406736(20)30566-3.

33. Gong J, Ou J, Qiu X, Jie Y, Chen Y, Yuan L, et al. A tool to early predict severe corona virus disease 2019 (COVID-19): a multicenter study using the risk nomogram in Wuhan and Guangdong, China. Clin Infect Dis. 2020. https://doi.org/10.1093/cid/ciaa443.

34. Imai Y, Kuba K, Neely GG, Yaghubian-Malhami R, Perkmann T, van Loo $\mathrm{G}$, et al. Identification of oxidative stress and Toll-like receptor 4 signaling as a key pathway of acute lung injury. Cell. 2008;133(2):235-49. https://doi.org/10.1016/j.cell.2008.02.043.

35. Sadik CD, Kim ND, Luster AD. Neutrophils cascading their way to inflammation. Trends Immunol. 2011;32(10):452-60. https://doi. org/10.1016/j.it.2011.06.008.

36. Amin Z, Rahmawati FN. Recent insight into potential acute respiratory distress syndrome. Saudi Med J. 2017;38(4):344-9. https:// doi.org/10.15537/smj.2017.4.15843.

37. McGonagle D, Sharif K, O'Regan A, Bridgewood C. The role of cytokines including interleukin-6 in COVID-19 induced pneumonia and macrophage activation syndrome-like disease. Autoimmun Rev. 2020;19(6):102537. https://doi.org/10.1016/j.autrev.2020. 102537.

Publisher's Note Springer Nature remains neutral with regard to jurisdictional claims in published maps and institutional affiliations. 